Material for the study: 637 sputum samples from 171 children of the intensive care unit, including from the department of pathology of newborns and premature infants; scrapes from the throat, nose and hands of 53 medical staff; 86 scrapes from the surfaces of the environment. Research methods: bacteriological and MALDI-TOF/MS (Bruker Daltonics).

It was found that 30 species of bacteria were isolated from children, including: Staphylococcus aureus (10\%), Staphylococcus epidermidis (8\%), Klebsiella pneumoniae (7\%), Streptococcus pneumoniae 7\%), Enterococcus faecium (2\%). S. aureus (45\%), S. epidermidis $(90 \%)$, S. pneumoniae $(17 \%)$, K. pneumoniae $(6 \%)$ were isolated from medical personnel serving these children. Staphylococci of different species with the highest proportion of $S$. epidermidis (21\%) were isolated from $50 \%$ of environmental objects. Most often they were isolated in newborns (16\%), less often in children aged 1 month to 1 year $(10 \%)$, in children $1-3$ years $(8 \%)$, in children older than 3 years $(1 \%)$. These data indicate a contact-household route of transmission of infection caused by $S$. epidermidis. The frequent occurrence of $S$. aureus and $S$. pneumoniae in children and medical personnel in the absence of these bacteria in the environment indicates the role of resuscitation department staff as a source of these infections. Bacteria $K$. pneumoniae and E. faecium are isolated from both children, medical personnel and environmental objects. All of the above bacteria were well subjected to destruction by disinfectants. After reorganization activities in the department, the proportion of positive bacterial seeding decreased significantly.

However, the study found that Bacillus cereus strains isolated from newborn infants $(6 \%)$ and from medical and general-purpose equipment are resistant to all disinfectants used in the department. As a result of the research, the drugs to which the isolated bacilli are sensitive were selected.

Thus, continuous monitoring of the microbiota, the study of its characteristics and the epidemiological approach to assessing the situation can significantly reduce or avoid the development of infections associated with the provision of medical care.

\section{9 doi: 10.15789/2220-7619-2018-4-1.9 \\ ETHICS OF VACCINATION AS THE CRITERIA OF THE SCIENTIFIC AND HUMANISTIC APPROACH}

\section{O.I. Kubar}

St. Petersburg Pasteur Institute, St. Petersburg, Russia

The aim of this research was to analyze the unique role of the vaccination from its successful and problematical sides concerning the developing, distribution and using the vaccines in different epidemiological situation in the mirror of the global bioethics. The role of the balance between ethics and success of vaccination was clearly illustrated and based on examples from the history of vaccination and our own experience connected with amazing contribution of Leningrad/St. Petersburg Pasteur Institute in the history of vaccination. It was clear shown that the particular importance has the harmonization and solidarity of all persons and structures involved in the process of vaccine prevention within local, national, regional, and global levels. In order to reach the goal in protection of the infections diseases by vaccination, it is necessary to follow in reality and to demonstrate to the society such universal ethical standards as scientific honesty, social expediency, justice, non-discrimination, transparency and overcoming of the conflict of interests. Detailed description how these ethical principals particularly work in the stage of $R \& D$ vaccines, its distribution, using for vulnerable population and in the frame of WHO program for eradication of poliomyelitis, measles, and rubella was done. The specific relevance of the presented study was to highlight the key role of ethical principles of the adversarial position to the increasing anti-vaccination lobby. It is well known that the success of vaccination is associated not only with effective and safe vaccines, but directly depends on the society adherence to vaccination acceptance and realization. A negative influence of anti-vaccination movement, which promotes mistrust to vaccination, is playing an essential role in this process. The objective analysis and identification of ethical errors in the process of vaccination demonstrated in this research show the way any unethical action is creating a basis for the anti-vaccination movement. The maintaining of ethical standards balance and building partnerships and interaction with society as well as the implementation the ethical elements in legal regulation of the vaccination process on national and international levels are crucial for achieving optimum results in countermeasures against evolving the anti-vaccination lobby and protection against infections by vaccination in the present time and future perspective.

1.10 doi: 10.15789/2220-7619-2018-4-1.10

\section{ETHICAL CONSIDERATION IN CONCEPTION OF INFECTIOUS DISEASES ERADICATION}

O.I. Kubar, M.A. Bichurina, N.I. Romanenkova, N.I. Rosaeva, N.V. Zheleznova, I.N. Lavrentieva, A.Yu. Antipova, O.I. Kanaeva

St. Petersburg Pasteur Institute, St. Petersburg, Russia

The goal of this study was to show the universal role of ethical conception in the realization WHO global initiative on the eradication of infectious diseases: polio, measles, rubella and congenital rubella. The analysis of the WHO Strategic Plan activities was done in the light of UNESCO Declaration "On Bioethics and Human Rights", 2005. During implementation of WHO Strategic Plan for eradication measles, rubella and congenital rubella at the national, regional and global levels was happened the obligatory need to twice postpone the deadline from 2010 to 2015 and from 2015 to 2020 . The reason of this event was connected with the lack of solidarity during preventive measures and clearly demonstrates the importance for joint actions and control over the epidemic process. The acceptation and following of these measures could help to achieve the effect, which corresponds to the ethical principals from article 13 "Solidarity and Cooperation" and article 24 "International Cooperation" of UNESCO Declaration. The diversity of situations and conditions in the implementation of the WHO program in different regions of the world, or in relation to the different cultural, social, religious, economic and psychological status of contingents requires adherence to the principles laid down in articles 8, 9, 12: "Recognition of human vulnerability and respect for personal integrity", "Equality, justice and equality" and "Respect for cultural diversity and pluralism". The whole system of administration and management of the WHO activity at each individual level of implementation should be based on the ethical principles decelerated in articles 3, 5, 14-17: "Human dignity and human rights", "Independence and individual responsibility", "Social responsibility and health", "Sharing benefits", "Protecting future generations" and "Protecting the environment, the biosphere and biodiversity." In general all elements of the implementation the global goals both in the field improving vaccine and vaccination pro- 